\title{
Impact of abiotic stresses on the protection efficacy of defence elicitors and on metabolic regulation in tomato leaves infected by Botrytis cinerea
}

Aljabal Maymoune 1

Email jaba178@yahoo.com

Picot Adeline 1,5

Email adeline.picot@univ-brest.fr

Turner Marie 1,*

Phone (+) 33298290644

Email turner@vegenov.com

Goulitquer Sophie 2,3

Email catherine.leblanc@sb-roscoff.fr

Charton Sophie 2,3,6

Leblanc Catherine 2,3

Neema Claire 4

Emailneema@supagro.inra.fr

Hallier Sonia 1,7

Emailssonia_hallier@yahoo.com

1 Vegenov-BBV, Penn ar Prat, 29250 St-Pol-de-Léon, France

2 Sorbonne Universités, UPMC University of Paris 06, UMR 8227, Integrative Biology of Marine Models, Station Biologique de Roscoff, CS 90074, 29688 Roscoff CEDEX, France

3 CNRS, UMR 8227, Integrative Biology of Marine Models, Station 
Biologique de Roscoff, CS 90074, 29688 Roscoff CEDEX, France AQ2

4 Montpellier Supagro, UMR BGPI - TA A-54/K, Campus International de Baillarguet, 34398 Montpellier Cedex 5, France

5 Present Address: Université de Brest, EA 3882, Laboratoire Universitaire de Biodiversité et Ecologie Microbienne, ESIAB, Technopôle BrestIroise, 29280 Plouzané, France

6 Present Address: Centre de Recherche Public Gabriel Lippmann, Département EVA, Plateforme de Protéomique et de Métabolomique Végétale, 4422 Belvaux, Luxemburg

7 Present Address: Kermorvan, 29410 Guiclan, France

\section{Abstract}

Finding sustainable plant protection strategies is a major challenge for agriculture. Taking advantage of the plant natural immune system by using plant defence elicitors is an interesting avenue to explore. However, transfer to field application is often difficult, mostly due to the complexity of interactions between plants and their environment, involving biotic and abiotic stresses. The protection efficacy against gray mold and the modes of action of potential elicitors were studied on tomato. Modulation of plant defense was studied using both global and targeted metabolic profiling. We identified seven potential elicitors showing good plant protection efficacy and able to trigger the oxylipin pathway, including jasmonic acid production, after inoculation with Botrytis cinerea. Following preliminary assays, seven elicitors including two well-studied elicitors (Bion 50WG ${ }^{\circledR}$ and BABA) showing good plant protection efficacy and low fungitoxic effect were selected to assay the effect of abiotic stresses (wounding, water stress and nitrogen deficiency) on their protection efficacy. Our results showed that the protection efficacy of all products was reduced when plants were exposed to abiotic stresses, suggesting an antagonistic interaction between the tomato responses to abiotic stresses and product treatments. We found that responses to leaf cuttings and product treatments induced metabolic changes in a time-dependent manner, and that both of which mainly activated the oxylipin and JA pathway. However, the negative effects of wounding on tomato protection efficacy of defence elicitors 
suggest that interplay with other antagonistic signalling pathways is also involved in the tomato responses to this combination of stress.

\section{Keywords}

Stress combinations

Wounding

Gray mold

Induced resistance

\section{Electronic supplementary material}

The online version of this article (doi: 10.1007/s10658-015-0606-y ) contains supplementary material, which is available to authorized users.

\section{Introduction}

Plant disease control in the 21 st century faces multiple challenges (Walters et al. 2005). Plant pathogens are continuously evolving, leading to the selection of strains able to circumvent host resistance genes or resistant to fungicides when repeatedly used. In addition, there are increasing concerns related to the environmental effects of widespread use of chemicals. In this context, alternative approaches of plant disease control need to be implemented in a sustainable way.

Plants naturally have the ability to defend themselves thanks to their immune system (Jones and Dangl 2006). Following perception of the pathogen by the plant, a complex signalling network takes place, in the centre of which the plant hormones salicylic acid (SA), jasmonic acid (JA), and ethylene (ET) are the key players (Sanchez et al. 2012). These signalling events ultimately lead to the establishment of plant defences, including the production of antimicrobial compounds and cell wall modifications. Among available biocontrol strategies, the induction of resistant mechanisms, notably through the application of plant defence elicitors (PDE), represents a promising alternative to chemical fungicides (Ballester et al. 2011). PDE can be defined as natural or synthetic compounds with no direct antifungal activity which, by mimicking natural signalling compounds, induce defence mechanisms in plants, in turn resulting in enhanced resistance to pests and pathogens upon attack (Fu and Dong 2013; Dufour et al. 2013). Alternatively, some compounds do not immediately trigger plant defence mechanisms but provide 
resistance through a faster and/or stronger induction of defence mechanisms following pathogen and pest infection. This phenomenon, named priming, should be preferred to strategies based on direct activation of plant defences because associated costs are reduced (Pastor et al. 2013). Since the end of the 80 s, plant treatments with elicitors were reported to induce defence mechanisms and allow broad spectrum resistance to pests and pathogens such as algal, microbial or plant extracts (Trouvelot et al. 2008; Klarzynski et al. 2000 ), plant hormones, their analogues or other chemicals (Cohen et al. 1994,2001; Métraux et al. 1990; Brisset et al. 2000), mycorhiza or microbial antagonists (Pozo and Azcón-Aguilar 2007). Since they generally have no toxic effects on the environment, elicitation or priming of plant defence may allow, in combination with other alternative solutions, effective and sustainable disease management in the field (Walters et al. 2005). AQ3

However, elicitors used in real field conditions often show a reduced efficacy when compared to results obtained under laboratory conditions, mainly because interactions with the environment affect the outcome of the induced resistance mechanisms. Indeed, plants are exposed to multiple biotic and abiotic stresses in combination rather than experiencing one stress at a time and they respond to such stresses through various signalling pathways that may interact or inhibit one another (Anderson et al. 2004; Asselbergh et al. 2008 ; Niinemets 2010). Such crosstalk may therefore interfere with PDE-induced resistance mechanisms and can partially account for the limited success of such a strategy under field conditions.

The fungus Botrytis cinerea is a plant necrotrophic pathogen responsible for gray mold on a broad range of crops, including grapevine, tomato, soft fruit and vegetable plants (Mansfield 1980). It is a major pathogen affecting tomato production worldwide and it causes severe yield losses in both preand post-harvest. Current control methods mainly rely on prophylactic practices, such as the disinfection of tools and greenhouses and the application of preventative fungicides. Elicitor treatments able to induce good protection efficacy against $B$. cinerea have been well documented, mainly under greenhouse conditions, including laminarin and chitosan in grapevine (Aziz et al. 2003, 2006), oligandrin in tomato (Lou et al. 2011), a Fusarium crude elicitor fraction on pepper (Veloso and Díaz 2013), oligogalacturonides from plant cell walls (Ferrari et al. 2007) and rhamnolipids (Sanchez et al. 2012 ) in Arabidopsis. However, to our knowledge, no study has yet focused 
on the performance of plant defence elicitors against $B$. cinerea when plants were subjected to various abiotic stresses. Notably, during tomato growth, leaf removal regularly performed by growers or insect attacks often results in wounded plants while nitrogen deficiency and/or water stress can also be of concern for field-grown tomatoes.

The aims of this study are: i) to determine the protection efficacy and the mode of action of 11 products of various origins including four well-known PDE: Bion 50WG, $\beta$-amino-butyric acid (BABA), Methyl jasmonate (MeJA) and Chitosan; ii) to evaluate the influence of abiotic stresses (wounding, nitrogen deficiency and water stress) on the protection efficacy of these agents; and iii) to better understand the plant physiological responses to a combination of abiotic and biotic stresses including leaf wounding, treatments with the products and inoculation with $B$. cinerea, using both global and targeted metabolic profiling by U-HPLC-MS/MS.

\section{Materials and methods}

\section{Plant material}

Experiments were done on tomato Lycopersicon esculentum (L.), using a variety (African) susceptible to $B$. cinerea. Plants were grown in semi-controlled greenhouse in pots containing compost until they reach a four-leaf stage, which approximately corresponds to 4 weeks after sowing. Plants were exposed to $14 \mathrm{~h}$ of light per day, temperature was set to $22 \pm 5{ }^{\circ} \mathrm{C}$ and hygrometry was not regulated. Every plant was watered with about $200 \mathrm{ml}$ every 4 days.

\section{Leaf treatments with products}

The products used in this study were dissolved at different concentrations in water as indicated in Table 1 and sprayed onto leaves using a fine glass atomizer (about $3 \mathrm{ml}$ per plant), 5 days prior to inoculation (see Online Resource, Fig 1). Based on literature, four agents for which the ability to induce plant defence mechanisms has been previously demonstrated were used here as positive "PDE controls": Bion $50 \mathrm{WG}{ }^{\circledR}, \mathrm{BABA}$, Chitosan and methyl jasmonate (MeJA) (Amborabe et al. 2004; Azami-Sardooei et al. 2013; Dufour et al. 2013; Eyre et al. 2006; Zhu and Tian 2012). In addition to those "PDE controls", the protection efficacy against $B$. cinerea of seven potential PDE were investigated (coded A to G; Table 1). A positive chemical reference, Rovral (see Table 1), was used in all experiments and always 
presented a protection efficacy of $100 \%$ (data not shown).

\section{Table 1}

List of products tested against Botrytis cinerea

\begin{tabular}{|c|c|c|}
\hline $\begin{array}{l}\text { Product } \\
\text { code }\end{array}$ & Active material and /or composition & Dosage \\
\hline A & $\begin{array}{l}\text { Coproduct of L-glutamic acid production by } \\
\text { fermentation }\end{array}$ & $0.5-1 \%$ \\
\hline $\mathrm{B}$ & Green tide algae Ulva sp. raw extract & $8 \%$ \\
\hline $\mathrm{C}$ & $\begin{array}{l}\mathrm{P} 2 \mathrm{O} 5(40 \% \mathrm{w} / \mathrm{v}) ; \mathrm{K} 2 \mathrm{O}(26.9 \% \mathrm{w} / \mathrm{v}) ; \mathrm{MgO} \\
(0.21 \% \mathrm{w} / \mathrm{v}) ; \mathrm{CaO}(0.21 \% \mathrm{w} / \mathrm{v})\end{array}$ & \\
\hline $\mathrm{D}$ & Saccharomyces cerevisiae extract & $0.15 \%$ \\
\hline $\mathrm{E}$ & $\begin{array}{l}\mathrm{P} 2 \mathrm{O} 5(40 \% \mathrm{w} / \mathrm{v}) ; \mathrm{K} 2 \mathrm{O}(26.9 \% \mathrm{w} / \mathrm{v}) ; \mathrm{MgO} \\
(0.21 \% \mathrm{w} / \mathrm{v}) ; \mathrm{CaO}(0.21 \% \mathrm{w} / \mathrm{v}) ; \mathrm{CaO} . \mathrm{SiO} 2 \\
(0.21 \% \mathrm{w} / \mathrm{v})\end{array}$ & \\
\hline $\mathrm{F}$ & Eugenol & $0.5 \%$ \\
\hline $\mathrm{G}$ & $\mathrm{KH} 2 \mathrm{PO} 3$ & $0.2 \%$ \\
\hline MeJA & Methyl Jasmonate & $800 \mathrm{mM}$ \\
\hline Chitosan & Chitosan & $2-4 \%$ \\
\hline Bion50WG ${ }^{\circledR}$ & $\begin{array}{l}\text { S-methyl benzo [1.2.3] thiadiazole-7-carbothioate } \\
\text { (BTH) }\end{array}$ & $0.08 \%$ \\
\hline $\mathrm{BABA}$ & b-amino-butyric acid & $15 \mathrm{mM}$ \\
\hline 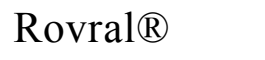 & Iprodione & $0.17 \%$ \\
\hline
\end{tabular}

\section{Plant inoculation with $B$. cinerea}

To prepare the spore suspension, $10 \mathrm{ml}$ of PDB (Potato Dextrose Broth, Conda Laboratory, Spain) and Tween ${ }^{\circledR} 80$ at $0.05 \%$ (Sigma-Aldrich, USA) were deposited on 7 day-old PDA culture of the strain DSM No. 877 (Strain designation : N51, Germany). The inoculum harvested from the plate was then filtered (using a sieve of $150 \mu \mathrm{m}$ mesh size) to separate the mycelium and spores. The suspension was then calibrated with a Malassez cell (Preciss, France), and the spore concentration was adjusted to $10^{3}$ spores $\mathrm{ml}^{-1}$. Plants were inoculated with implants of $B$. cinerea 5 days after treatments with each product (see Online Resource, Fig 1). In order to prepare the implants, $1 \mathrm{ml}$ of spore suspension was deposited on a new PDA plate. Thirty minutes later, a $4 \mathrm{~mm}$ diameter piece was cut and deposited on the top of each of the first two 
leaves (spores contact the leaf). Leaves were previously sprayed with water to promote the adhesion of the implants. Two to four leaflets per leaf of each plant were inoculated. After inoculation, plants were placed in trays in the growth cabinet calibrated to $25 \pm 5{ }^{\circ} \mathrm{C}$ at night $/ 25 \pm 5{ }^{\circ} \mathrm{C}$ during the day; HR $>85 \%$, and $18 \mathrm{~h}$ of darkness. The trays were covered with a plastic cover for $18 \mathrm{~h}$ to favour spore germination. Disease evaluation was carried out 5 days after inoculation by measuring the length of the necrosis caused by $B$. cinerea (see Online Resource, Fig 1).

\section{Experimental design}

The assays were organized into four technical replicates. Each replicate contained the plants treated with each of the products randomly distributed, with one to two plants per tested product. Two to three independent biological experiments were carried out depending on the product.

For each experiment, the protection efficacy (PE) of tested products was calculated compared to water-treated controls and as the mean of replicates. For each replicate i (rep i), PE was calculated as follows: PE rep i $(\%)=$ [(mean necrosis length on water-treated control in rep $\mathrm{i}$ - mean necrosis length on product-treated plants in rep i) / mean necrosis length on watertreated control inrep i] $\times 100$.

\section{Biocide effect of tested products on spore germination and mycelial growth}

Five-hundred $\mu 1$ of a fresh spore suspension at $4 \times 10^{3}$ spores $\mathrm{ml}^{-1}$ were mixed with $500 \mu 1$ of each product (diluted either at the concentration used in the disease assay or ten times this concentration), sprayed onto PDA and incubated at $20^{\circ} \mathrm{C}$ for $17 \mathrm{~h}$. The percentage of germinating spores was evaluated and compared to that found in water treated controls (i.e., when spores were mixed with $500 \mu \mathrm{l}$ of sterilized water). Mycelial growth was examined as well. $80 \mu \mathrm{l}$ of each tested product were placed on two pads of sterile filters, placed on the edge of the PDA dishes, and then an implant of spores was placed in the middle of the dishes at room temperature. The fungicidal effect was evaluated by measuring the colony diameter of $B$. cinerea 3 days after inoculation. Each assay was arranged in four technical randomized replicates (i.e., four dishes). At least two independent biological experiments were carried out per assay.

\section{Application of abiotic stresses}


The impact of three abiotic stresses (water stress, nitrogen deficiency and wounding) on the protection efficacy of nine agents (Products A-G and BABA and Bion 50WG ${ }^{\circledR}$ as PDE controls) was studied. For all abiotic stresses, plants were treated, inoculated and scored for disease development as described before. Two independent biological experiments were carried out.

Wounding the apical portion of each leaflet of stressed plants was cut off (see Online Resource, Fig 1) 2 days before treatment. To determine whether timing of wounding influences the protection efficacy of the products, a comparison with wounding applied after treatment or just prior inoculation, was also performed for products A, E and PDE controls.

Water stress All tested plants were irrigated with $200 \mathrm{ml}$ of water per plant every 4 days, from sowing until 1 week before treatment with products. Irrigation of stressed plants was stopped 1 week before treatment. After treatment, stressed plants were irrigated with $10 \mathrm{ml}$ of water every 2 days until scoring (see Online Resource, Fig 1). Irrigation of the non-stressed plants was maintained with $200 \mathrm{ml}$ of water per plant every 2 days from sowing until disease evaluation.

Nitrogen deficiency Two nutritive solutions, at 100 and $7.5 \%$ of nitrogen requirements, were prepared to feed plants grown in vermiculite. The complete nutritive solution (100 \%) contained: $\mathrm{Ca}(\mathrm{NO} 3) 2: 9.7 \mathrm{~g}, \mathrm{KNO} 3$ : 5.3 g, MgSO4: 4.9 g, KH2PO4: 2.5 g, Fer EDDHA: 225 mg, OligoMix: 56 mg in 101 of water. The solution used for nitrogen deficiency (7.5\%) contained: Ca(NO3)2: 0.5 g, MgSO4: 4.9 g, KCl: 3.8 g, CaCl2: 4.1 g, Fer EDDHA: $225 \mathrm{mg}$, OligoMix: $56 \mathrm{mg}$ in 101 of water. $200 \mathrm{ml}$ per plant of the complete and deficient solutions were respectively applied to unstressed and deficient plants every 4 days, from sowing until disease scoring.

\section{U-HPLC-MS/MS metabolite profiling}

For metabolic profiling studies, plants were treated with water as negative control, and Bion ${ }^{\circledR}$ and BABA as PDE controls and inoculated with $B$. cinerea as previously described. Leaf samples were collected at four time points: 5 days before inoculation ( $5 \mathrm{dbi}$ ), just prior treatment (3dbi), the day of inoculation, i.e., just prior inoculation (d0) and 4 days after inoculation (4dpi), when necrosis was large enough (see Online Resource, Fig 1). The $3 \mathrm{~mm}$ part surrounding the necrotic region was harvested (see Online Resource, Fig 1). In addition to analyzing PDE controls, we analyzed the 4dpi metabolic.. 
profiling of samples treated by products A, B, D, E and G.

To explore the mechanisms of action of putative PDE in inducing resistance against $B$. cinerea and those responsible for the loss of efficacy in pre-stressed plants, we chose to conduct global and targeted metabolomic studies using U-HPLC-MS/MS on tomato plant leaves, under wounding stress, pre-treated or not with different putative PDE before infection with $B$. cinerea. Bion $50 \mathrm{WG}{ }^{\circledR}$ and $\mathrm{BABA}$ were first used as PDE positive controls, to study the combined effects of wounding and PDE-treatments during a time-course. The tomato metabolic profiling were established in samples collected 2 days after cutting (i.e., 5 days before inoculation with $B$. cinerea ( $5 \mathrm{dbi}$ ), and just before PDE-treatment), 3 days before inoculation ( $3 \mathrm{dbi}$ ), just before inoculation (d0) and 4 days after B. cinerea inoculation (4dpi) (see Online Resource, Fig 1). Using all the metabolic data, a principal component analysis (PCA) was performed to analyze the relationships between the three variables (i.e., PDE-treatment, wounding stress and sampling time). Each sample was obtained from a pool of four leaflets from two different plants per sampling date, and the experiments contained three technical replicates. After being collected, samples were immediately frozen in liquid nitrogen, freeze-dried and stored at $-20^{\circ} \mathrm{C}$. Samples were ground in CK-Mix tubes using a Precellys grinder (Bertin Technologies, Montigny-le-Bretonneux). Extraction was carried out by adding $1 \mathrm{ml}\left[\mathrm{MeOH} / \mathrm{H}_{2} \mathrm{O}(8: 2)\right]$ containing $1.25 \mu \mathrm{g} 12-\mathrm{OH}-$ Lauric acid as internal standard and by shaking for $1 \mathrm{~h}$ at $4{ }^{\circ} \mathrm{C}$. Extracts were centrifuged for $10 \mathrm{~min}$ at $17500 \times \mathrm{g}$ at $4{ }^{\circ} \mathrm{C}$ and supernatant was transferred in a glass vial stored at $-80{ }^{\circ} \mathrm{C}$ until analysis. Sample fingerprinting was performed on a Dionex Ultimate 3000 RSLC system including an autosampler, a tertiary pump and coupled to a Finnigan LTQ-Orbitrap ${ }^{\text {TM }}$ hybrid mass spectrometer (Thermo Fisher Scientific, Bremen, Germany). Chromatographic separation was performed on an Acclaim 120 C18 column $(100 \times 2.1 \mathrm{~mm} \times 2.2 \mu \mathrm{m}$ particle size, Dionex $)$. Mobile phase consisted in water containing $0.1 \%$ acetic acid (A) and acetonitrile containing $0.1 \%$ acetic acid (B). The used elution gradient (A:B, v/v) was as follow: 80:20 from 0 to $5 \mathrm{~min}$; $95: 5$ at $15 \mathrm{~min}$ and hold for $10 \mathrm{~min}$; 80:20 at $26 \mathrm{~min}$ and hold for $4 \mathrm{~min}$. The injected volume was $5 \mu \mathrm{l}$, the flow rate was $0.25 \mathrm{ml} \mathrm{min}^{-1}$ and the temperature of the column was maintained at $20{ }^{\circ} \mathrm{C}$. The UHPLC column was connected without splitting to the electrospray interface operating in the positive ion mode. The electrospray voltage was set to $3.5 \mathrm{kV}$, the capillary voltage to $45 \mathrm{~V}$, and the tube lens offset to $130 \mathrm{~V}$. The sheath and auxiliary gas flows (both nitrogen) were set to five arbitrary units (a.u.), and the drying gas 
temperature was set to $300{ }^{\circ} \mathrm{C}$. Mass spectra were recorded from 50 up to $1000 \mathrm{~m} / \mathrm{z}$ at a resolution of 30000 (FWHM at $\mathrm{m} / \mathrm{z} 400$ ). Mass spectra were acquired in the centroid mode. For data processing, following their acquisition by Xcalibur ${ }^{\circledR}$ software (Thermo Fisher Scientific), metabolomic fingerprints were deconvoluted to allow the conversion of the three-dimensional raw data ( $\mathrm{m} / \mathrm{z}$, retention time, ion current) to time- and mass-aligned chromatographic peaks with associated peak areas. Massmatrix File Conversion was used to convert the original Xcalibur data files (*.raw) to a more exchangeable format (*.mzXML). Data processing was then performed using the open-source XCMS software. XCMS parameters for the R language were implemented in an automated script. CentWave was used for the peak picking. The interval of $\mathrm{m} / \mathrm{z}$ value was set to 0.1 , the signal to noise ratio threshold was set to 10 , the group band-width was set to 10 and the minimum fraction was set to 0.75 . SIMCA 13.0 (Umetrics, Malmö, Sweden) was used for statistical analysis. Identification of the different metabolites was carried out using the Metlin database and in-house standards. Xcalibur was later used for the quantification of chosen metabolites.

\section{Statistical analyses}

Statistical analysis was performed using Splus software v.6.1 (TIBCO Sofware Inc., USA) for ANOVA and multiple comparisons and using R v2.15.0 ( http://www.r-project.org ) for Principal Component Analysis (PCA). Targeted metabolite data was $\log 10$-transformed as $\log 10(13-\mathrm{HpODE}+0.01)$, $\log 10(13-\mathrm{HOTrE}+0.1), \log 10(\mathrm{JA}+0.001)$ and $\log 10(\mathrm{SA}+0.001)$ to ensure normal distribution and homogeneity of variance. Differences between treatments were determined with multiple mean comparisons with the control's mean (e.g., water treatment) using the simulation-based method (determined by software as the best procedure with the smallest critical point among all valid methods). For the protection efficacy assays, a Bonferroni adjustment was used and the level of significance for multiple comparisons between treatments was set at $p<7,57 \times 10^{-4}(0.05 / 66$ combinations; Fig. 1$)$.

\section{Fig. 1}

Protection efficacy of seven putative PDE or PDE references against gray mold development on tomato. Mean necrosis length measured 5 days after inoculation with $B$. cinerea (a) on tomato plant treated with water, seven putative PDE or PDE references. Protection efficacy of these putative PDE (A-G, white bars) was evaluated and compared to water-treated controls (b). Four reference alternative products (dashed bars): Methyl Jasmonate (MeJA), BABA, Bion 
50WG and Chitosan were included. Standard deviation was calculated on two to three independent experiments. Differences between treatments were determined with multiple comparisons using the Bonferroni adjustment ( $\alpha=$ $\left.7,57 \times 10^{-4}\right)$
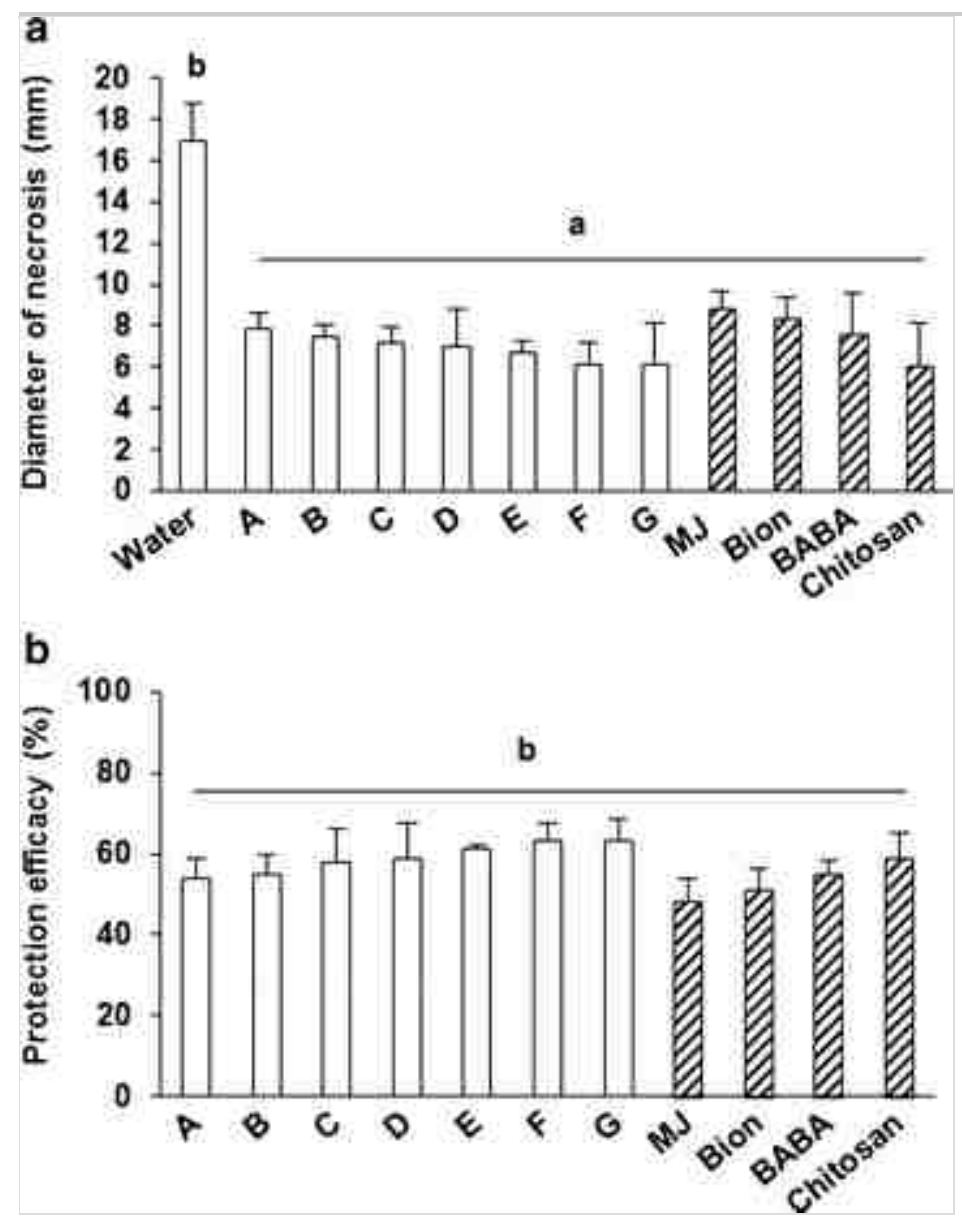

\section{Results}

\section{Identification of potential PDE efficient against tomato gray mold}

We investigated the protection efficacy against $B$. cinerea of four well-known PDE and seven potential PDE (coded A to G, Table 1). The mean length of necrosis in water-treated controls was $16 \mathrm{~mm} \pm 2.2$ (Fig. 1a). Treatments with the four PDE controls induced a significant smaller necrosis length compared to water-treated plants $\left(p\right.$-value $\left.=10^{-6}\right)$, (Fig. 1a) with the following protection efficacy: Chitosan (59\%), BABA (55\%), Bion ${ }^{\circledR}(51 \%)$ and MeJA (48 \%) (Fig. 1b). Each of our potential PDE was also able to significantly reduce disease development compared to water-treated plants with the following protection efficacy: A (54\%), B (55\%), C (58\%) D (59\%), E $(61 \%), \mathrm{F}(63 \%)$ and $\mathrm{G}(63 \%)$, ( $p$-value $\left.=10^{-6}\right)$. The fungicidal effect of all products was then evaluated at two doses (the dose used in the disease assay 
and ten times this dose, below refer to as dose $1 \times$ and $10 \times$ ) on both mycelial growth of $B$. cinerea and spore germination, in comparison to water-treated controls (with a mean mycelial growth of $40 \mathrm{~mm} \pm 1.5,3$ days post-inoculation and a spore germination rate of $100 \%$ ). No fungicidal effect on mycelial growth was observed with any of the products whatever the concentration tested, when compared to water-treated controls ( $p$-value $=$ 0.14). Regarding the impact on spore germination (Fig. 2), Chitosan among the four PDE induced a significant effect, compared to water-treated controls with $25 \%$ of spore germination inhibition at dose $10 \times\left(p\right.$-value $\left.=5.10^{-6}\right)$. In addition, four products ( $\mathrm{A}$ and $\mathrm{C}$ at the two doses; $\mathrm{B}$ and $\mathrm{G}$ at dose $10 \times$ ) showed a significant negative effect on $B$. cinerea spore germination but at a lower level than chitosan (percentage of spore germination inhibition ranging from 7 to $10 \%)\left(p\right.$-value $\left.=10^{-6}\right)($ Fig. 2$)$. The other products did not show any significant effect on spore germination at the two doses tested $(p$-value $=$ $0.1)$.

\section{Fig. 2}

B. cinerea spore germination inhibition caused by seven putative PDE and PDE references, tested at two doses. Seven products and four reference alternative products (Methyl Jasmonate (MeJA), BABA, Bion 50WG and Chitosan) were tested. Two product doses were assayed, $1 \times($ white bars $)$ and $10 \times($ black bars $)$. Germination inhibition of products was calculated as a ratio of germinated spores in water control. Standard deviation was calculated on two independent experiments. The star indicates significant differences with the water-treated controls, for which $100 \%$ of the spores germinated (ANOVA, $\alpha=0.05$ )

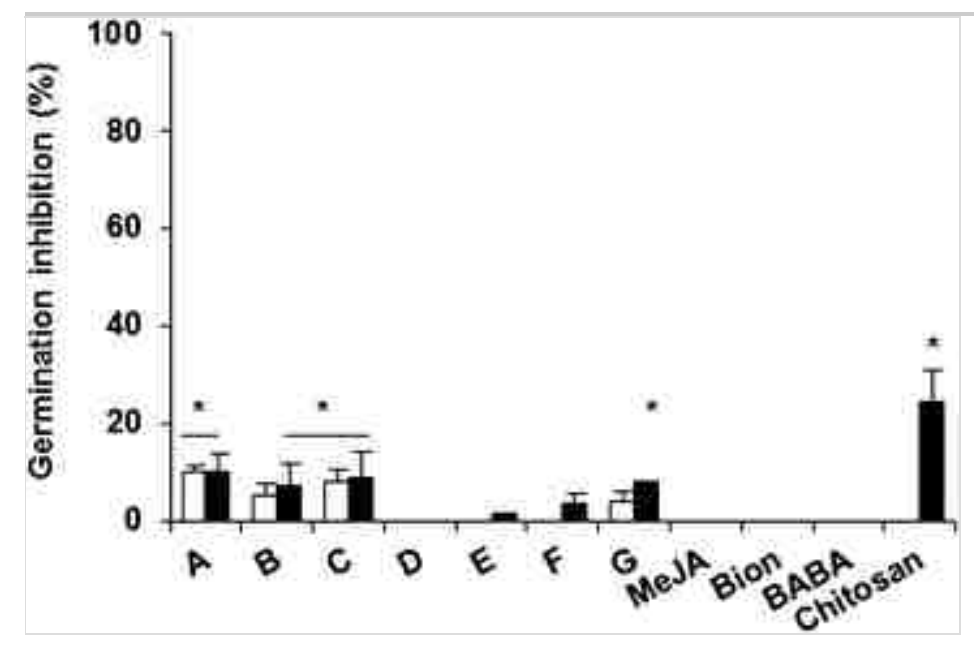

\section{Influence of abiotic stress on the protection efficacy of PDE}

The influence of abiotic stress on the protection efficacy of PDE was 
examined for three types of stress: leaf wounding, water stress and nitrogen deficiency.

First, the effects of abiotic stresses were evaluated on disease development in plants treated with water. Necrosis lengths caused by $B$. cinerea were always higher on plants previously exposed to an abiotic stress when compared to non-stressed plants, with significant differences found for water stress and nitrogen deficiency ( $p$-value $=0.0081,<0.0001$ and 0.0881 for water stress, nitrogen deficiency and wounding, respectively) (Fig. 3). Under our experimental conditions, the ability of $B$. cinerea to colonize the host was therefore enhanced on pre-stressed plants.

\section{Fig. 3}

Mean necrosis length 5 days after inoculation with $B$. cinerea on tomato watertreated plants previously stressed or not and treated with water. The histograms (white: stressed; black: non-stressed plants) represent the mean of three independent assays for the wounding experiment and two assays for water stress and nitrogen deficiency experiments. The bar represents the standard deviation of the two or three independent experiments. The star indicates if there is significant difference between pre-stressed plants infected with $B$. cinerea and plants only infected with $B$. cinerea (ANOVA, $\alpha=0.05$ )

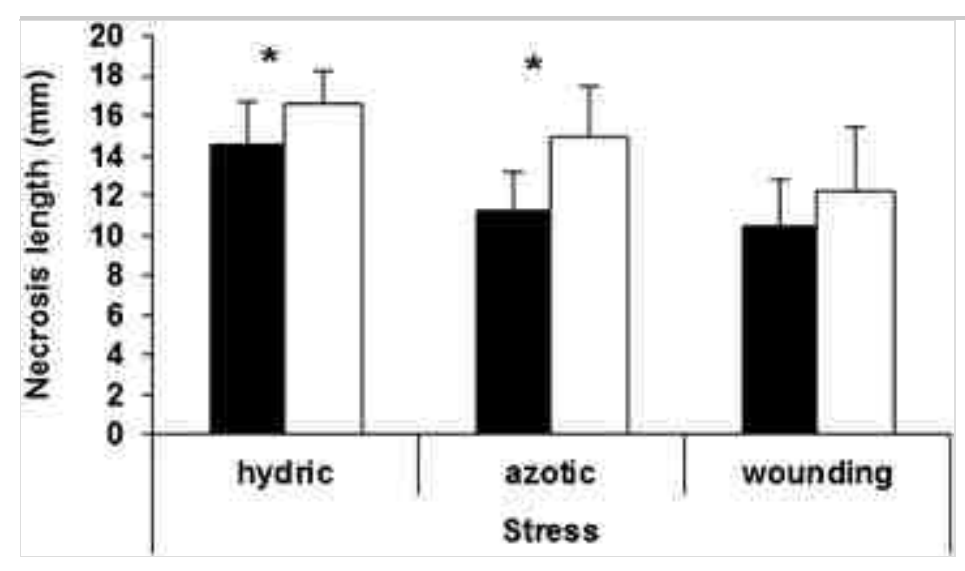

We then studied the influence of abiotic stresses on the efficacy of two PDE controls (BABA and Bion) and the products A-G (Fig. 4). Whatever the nature of the abiotic stress, the ANOVA indicated a significant effect of both the products ( $p$-value $=10^{-6}$, for water stress, nitrogen deficiency and wounding, respectively) and the application of the abiotic stress or not ( $p$-value $=3.10^{-5}, 10^{-6}$ and $10^{-6}$, for the three types of stress, respectively) on the necrosis length with no significant two-way interaction. In addition, whatever the nature of the abiotic stress, the protection efficacy of the 
products was always lower on pre-stressed plants compared to non-stressed plants, with significant differences for most of the products (Fig. $4 \mathrm{a}-\mathrm{c}$ ). The reduction in protection efficacy between pre-stressed and non-stressed plants ranged from 9 to $27 \%$, depending on the product and the nature of the abiotic stress. Overall, our results clearly highlight that protection by PDE and potential PDE is affected in plants which have been previously exposed to wounding, nitrogen deficiency or water stress. Note however that treatments with the products were still able to significantly reduce disease development on pre-stressed plants compared to water-treated controls $(p$-value $=0.001)$.

\section{Fig. 4}

Protection efficacy of seven putative PDE and two PDE references against gray mold development. Protection efficacy of seven selected products $(A-G)$, and PDE references products (Bion $50 \mathrm{WG}{ }^{\circledR}$ and BABA) were assayed by measuring disease development 5 days after inoculation and compared to the protection efficacy observed in water-treated plants inoculated with $B$. cinerea Plants were treated 5 days before inoculation ( $5 \mathrm{dbi}$ ) and were either stressed (white and gray bars) or not (black bars). Water stress (a), nitrogen deficiency (b) and wounding (c and d) were studied. Wounding was either applied 2 days before treatment (i.e., 7 days before inoculation) (c), or at two different time-points, 2 days before treatment (white bars) or at the moment of inoculation (gray bars) (d). Standard deviation was calculated on two independent exepriments. Stars indicate significant difference between non-stressed and pre-stressed plants (ANOVA, $\alpha=0.05$ ). Dashes above the histograms indicate that no significant difference (c) 

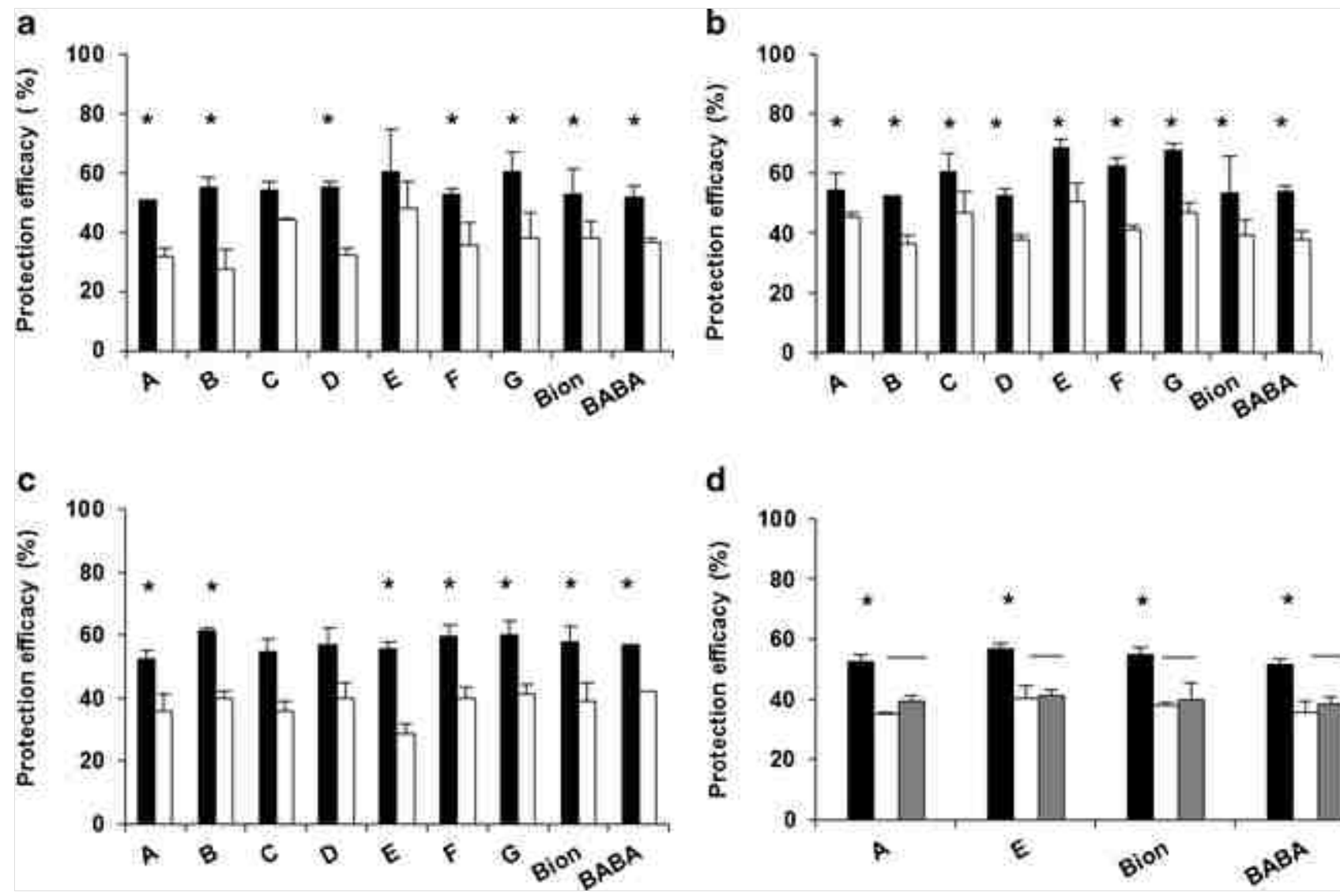

The timing of wounding effect was also studied: plant were stressed, either 2 days before treatment (i.e., 7 days before inoculation), or just prior to the inoculation (d0), (see Online Resource, Fig 1) with no significant differences in disease development. Both timing of wounding led to a similar loss of efficacy, whatever the product tested (Fig. 4d).

Studies of tomato metabolic regulation under wounding, PDE treatments and $B$. cinerea infection

The tomato metabolic profiling was established in samples collected as previously mentioned at four sampling times. Using all the metabolic data, a principal component analysis (PCA) was performed to analyze the relationships between the three variables, (i.e., PDE-treatment, wounding stress and sampling time). The plots showed that the first (16\%) and second components of the total variance (12\%) for all the pooled data were mostly explained by the sampling time, and in a smaller proportion by the wounding pressure, but not by the PDE-treatments (Fig. 5). Because of the strong effect of sampling time, PCA were then performed independently at each time point to analyze the relationships between PDE-treatment (Product) and wounding stress on metabolic responses (see Online Resource, Fig. 2). These statistical analyses showed that before infection (at $5 \mathrm{dbi}$ and $3 \mathrm{dbi}$, see Online Resource, Fig. 1), the most important loadings on the first component (respectively, 22 
and $17 \%$ ) were the Wounding and Control variables, whereas 5 days after PDE-treatment ( $\mathrm{d} 0$ ) and moreover 4 days after inoculation by $B$. cinerea (4dpi) the two first components of the total variance were better explained by the PDE-treatments (Product variable), especially by the BABA-treatment, than by stress application (Treatment variable) (see Online Resource, Fig. 2).

\section{Fig. 5}

Principal Component Analysis (PCA) on metabolic profiling of tomato leaves harvested at different times, after wounding, PDE-treatments and B. cinerea inoculation. The PCA was performed on 71 samples, described by 130 metabolites and three qualitative variables (Product: water, Bion WG ${ }^{\circledR}, \mathrm{BABA}$; Time: 5 dbi, 3 dbi, d0, 4 dpi; Stress: Wounding, Control); (see Online Resource, Fig. 1)

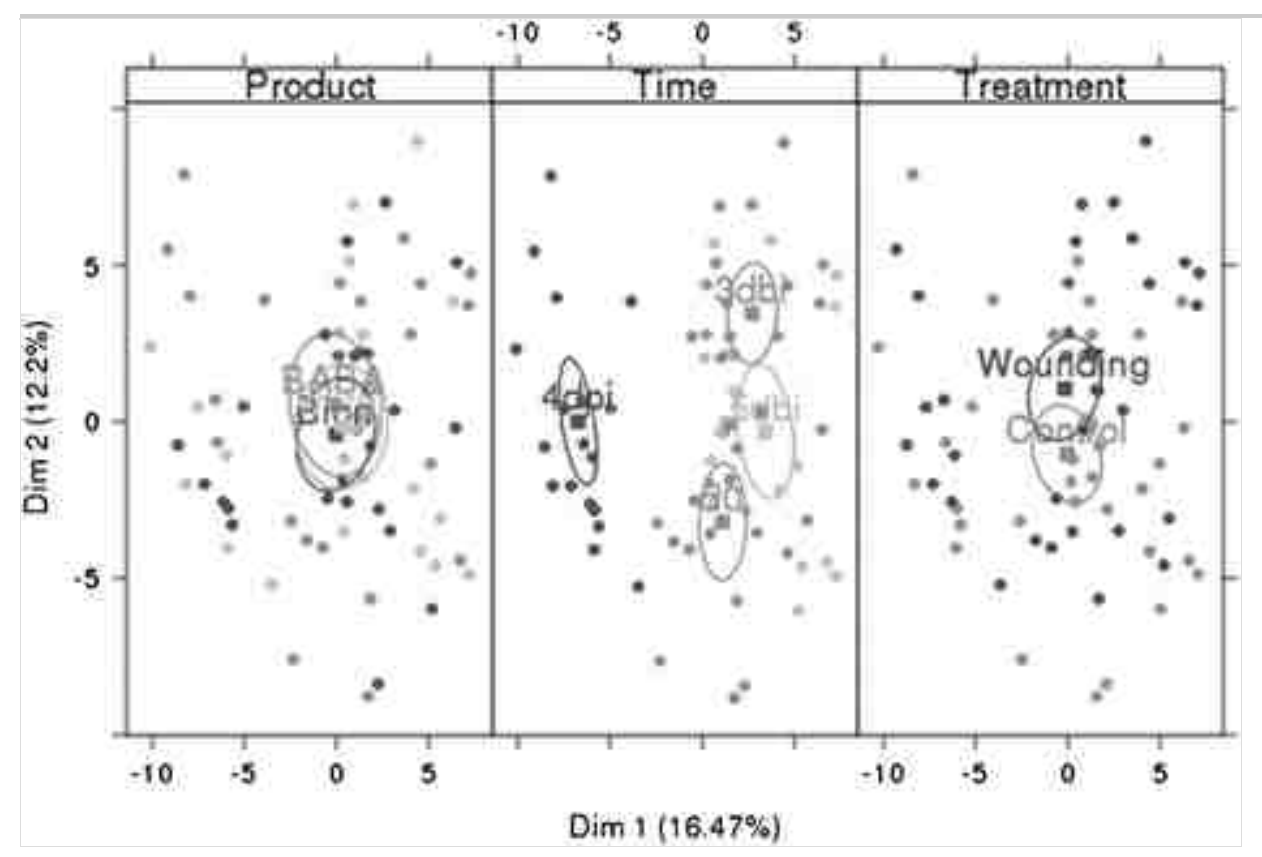

To study the effect of wounding on metabolic regulation, we specifically analyzed the metabolic responses of tomato leaves, harvested 2 days after wounding (5dbi). When compared to non-stressed leaves, 10 upon the first 15 metabolites shown to feature significant increases in all wounded leaves ( $p$-value $\leq 0.024 ; 1.1$ to 3.6 fold) were identified as oxylipins (see Table 2; Online Resource, Table 1). Moreover the metabolic profiling of these 10 oxylipins strongly discriminated control and wounded samples in a partial least squares discriminant analysis (PLS-DA) (see Online Resource, Fig. 3 ). 


\section{Table 2}

List of the 10 metabolites, significantly up-regulated in wounded leaves compared to control plants, harvested 5 days before inoculation and 2 days after wounding

\begin{tabular}{|l|l|l|l|l|}
\hline $\begin{array}{c}\text { XCMS } \\
\text { name }\end{array}$ & $\begin{array}{c}\text { Fold } \\
\text { change }\end{array}$ & P-value & Formula & Metlin ID \\
\hline M293T11 & 2.1 & 0.001 & C18H30O3 & 13-HOTrE \\
\hline M235T11 & 3.4 & 0.001 & C15H24O2 & $\begin{array}{l}\text { Fragment from } \\
\text { Octadecatetraenoic acid }\end{array}$ \\
\hline M265T11 & 3.6 & 0.002 & C16H26O3 & Fragment from 13-HODE \\
\hline M295T12 & 1.7 & 0.002 & C18H32O3 & 13-HODE \\
\hline M275T11 & 1.7 & 0.003 & C18H28O2 & Octadecatetraenoic acid \\
\hline M593T11 & 2.0 & 0.006 & C27H47O12P & PI(18:3/0:0) \\
\hline M407T24 & 1.9 & 0.007 & C19H35O7P & $\begin{array}{l}\text { Palmitoylglycerone } \\
\text { phosphate }\end{array}$ \\
\hline M291T12 & 1.6 & 0.008 & C18H28O3 & 12-oxo-PDA \\
\hline M433T24 & 1.3 & 0.017 & C21H39O7P & Lysophosphatidic acid \\
\hline M431T24 & 1.1 & 0.024 & C26H50NO7P & $\begin{array}{l}\text { 1-linolenoyl-sn-glycero- } \\
\text { 3-phosphate }\end{array}$ \\
\hline
\end{tabular}

In order to further study resistance mechanisms against necrotrophic pathogens, we also performed a time-course metabolite profiling of jasmonic acid (JA), salicylic acid (SA) and two targeted oxylipins, 13-hydroperoxyoctadecadienoic acid (13HpODE), 13-hydroxy-octadecatrienoic acid (13HOTrE), chosen as marker of these defence reactions. Production levels of the four compounds were compared between inoculated plants treated with PDE products (A, B, D, G, Bion, BABA) or with water (Table 3). The A, B, $D$ and $G$ products were selected because they showed good protection efficacy and relatively low direct effect. Significant increases of 13HpODE and/or JA compared to water control were detected at $4 \mathrm{dpi}$ for all treatments while no significant differences of $13 \mathrm{HOTrE}$ levels were found between the water treatment and the product treatments (Table 3 ). In our conditions, BABA mainly repressed or did not modulate the production of the selected metabolites (ratios mainly ranging between 0 and 1), with the exception of a significant increase of 13-HpODE at 4dpi (Table 3). Regarding SA, increased levels (over 2-fold changes) were found in Bion-, A-, B- and D-treated leaves 
compared to water control at $\mathrm{d} 0$ and $4 \mathrm{dpi}$, with significant differences only detected in Bion-treated leaves at d0. Significant decreased levels of SA were found in G-treated leaves at 4dpi (Table 3 ).

\begin{tabular}{|c|c|c|c|c|c|}
\hline \multirow{2}{*}{ Products } & \multirow{2}{*}{ Time } & \multicolumn{4}{|c|}{ Metabolites } \\
\hline & & 13-HрODE & 13-HOTrE & JA & SA \\
\hline \multirow{3}{*}{ BION50WGß } & $3 \mathrm{dbi}$ & 5.3 & 2.4 & ND & 1.9 \\
\hline & $\mathrm{d}^{*}$ * & 0.2 & 0.4 & 0.9 & 9.0 \\
\hline & 4dpi & 9.3 & 1.8 & 7.4 & 2.7 \\
\hline \multirow{3}{*}{ BABA } & $3 \mathrm{dbi}$ & 3.6 & 2.3 & ND & 1.3 \\
\hline & d0 & 0.5 & 0.4 & 0 & 1.1 \\
\hline & $4 \mathrm{dpi}$ & 2.8 & 0.6 & 0 & 0.5 \\
\hline \multirow{3}{*}{ G } & $3 \mathrm{dbi} *$ & 4.8 & 0.5 & ND & 1.5 \\
\hline & $\mathrm{d}^{*}$ * & 0.9 & 0.4 & 3.1 & 1.0 \\
\hline & 4dpi * & 5.3 & 1.3 & 2.2 & 0.1 \\
\hline \multirow{3}{*}{ D } & $3 \mathrm{dbi}$ & 19.0 & 1.2 & ND & 2.9 \\
\hline & do & 3.2 & 0.6 & 1.3 & 2.8 \\
\hline & 4dpi & 16.9 & 1.7 & 11.7 & 3.5 \\
\hline \multirow{3}{*}{ A } & $3 \mathrm{dbi} *$ & 2.9 & 1.3 & ND2 & 1.6 \\
\hline & do * & 0,6 & 0.7 & 7.1 & 3.2 \\
\hline & 4dpi* & 7.4 & 1.3 & 4.9 & 1.9 \\
\hline \multirow{3}{*}{ B } & $3 \mathrm{dbi}$ & 16.5 & 0.6 & ND & 1.7 \\
\hline & do & 1.3 & 0.3 & 3.0 & 2.0 \\
\hline & $4 \mathrm{dpi}$ & 8.3 & 2.1 & 4.3 & 2.3 \\
\hline
\end{tabular}

\section{Table 3}

Relative metabolic ratio in tomato leaves pre-treated with PDE controls and putative PDE, compared to water-treated controls

Leaves were harvested 2 days after treatment, 3 days before inoculation ( $3 \mathrm{dbi}$ ) with $B$. cinerea, at the time of the inoculation $(\mathrm{d} 0)$ and 4 days post inoculation (4 dpi). Two PDE controls (BION 50WG ${ }^{\circledR}$ and BABA) and four putative PDE (A, B, D and G) were tested. Metabolites were either not significantly induced (white), significantly induced (gray) or significantly repressed (black)

ND not detected in both product-and water-treated samples. ND2 not detected in water-treated samples but detected in A-treated samples. Values are mean of fold changes in product-pretreated samples compared to water-treated samples $(n=3 ; *$ : $n=2)$. 13-HpODE 13-hydroperoxy-octadecadienoic acid (oxylipin; fatty acid oxygenated), 13-HOTrE 13-hydroxy-octadecatrienoic acid (fatty hydroxyl), $J A$ Jasmonic Acid, $S A$ Salicylic acid

Finally, we also investigated the action mode of new potential PDE (A, B, D, and $\mathrm{E}$ ) and analyzed the metabolic profiling of tomato leaves harvested 4 days after inoculation by $B$. cinerea, following wounding and PDE treatment. 
Using PCA, the global metabolic responses were compared between control and wounded plants, and non-inoculated (nic) and inoculated plants after the different PDE-treatments (including Bion and BABA) (Fig. 6). As previously shown for water, Bion and BABA treatments (see Online Resource, Fig. 2), at 4dpi, the Wounding/Control variables (Treatment) were not significantly different, while some Product variables were important loadings on the first two components of the total variance. The non-inoculated plant profiles (nic) appeared well separated from all inoculated tomato samples. Some PDE-treated plant metabolic profiling, e.g., with B, D, and Bion, were not significantly distinct from the water-treated samples. On this first axis of the total variance (18\%), A- and BABA-treated samples were the most important loadings, whereas on the second axis (12\%), it was the E-treated plants (Fig. 6).

\section{Fig. 6}

Principal Component Analysis (PCA) on metabolic profiling of tomato leaves harvested 4 days post-inoculation with $B$. cinerea and previously submitted to wounding and different PDE-treatments. The PCA was performed on 48 samples, described by 130 metabolites and two qualitative variables (Product: nic, A, B, D, E; water, Bion, BABA; Stress: Wounding, Control). nic non-inoculated control; (see Table 1)

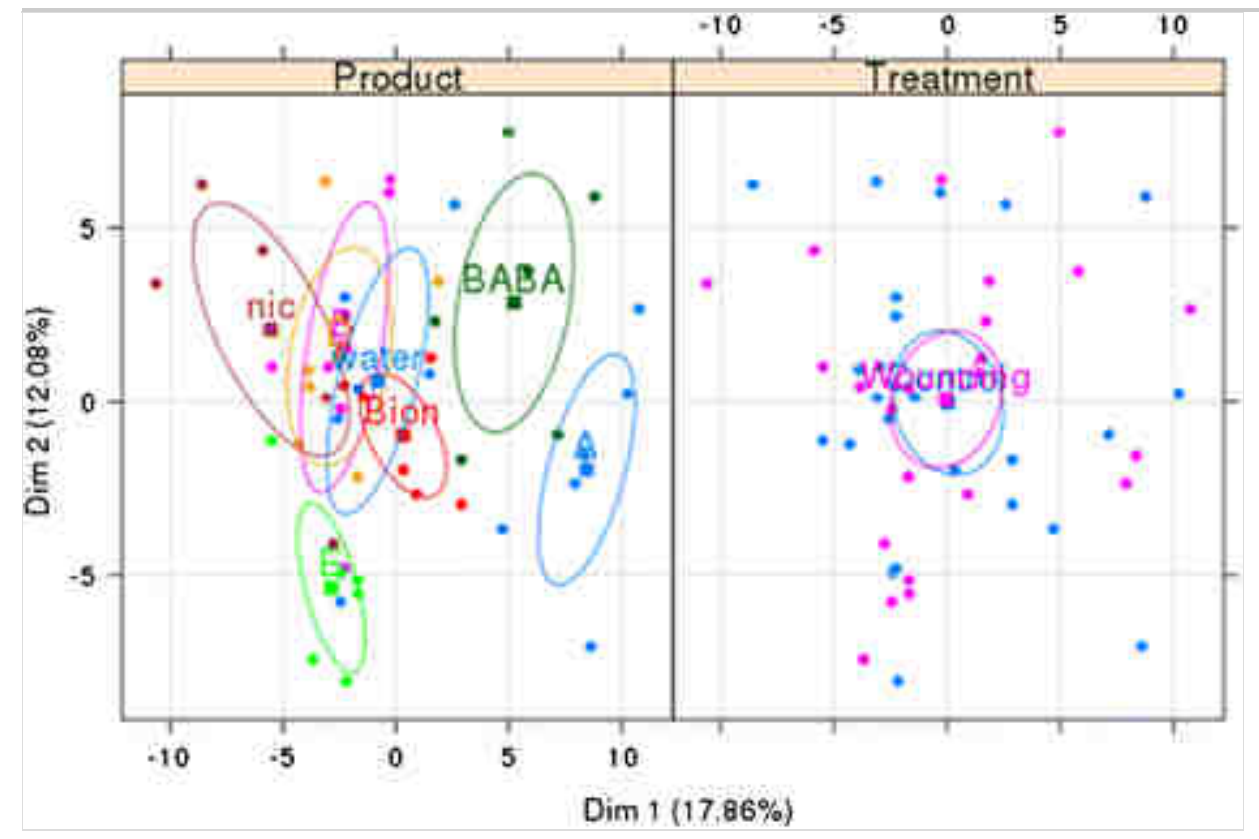

\section{Discussion}

In this paper, we studied the protection efficacy of four established PDE (Bion $50 \mathrm{WG}{ }^{\circledR}, \mathrm{BABA}, \mathrm{MeJA}$, and Chitosan) against $B$. cinerea and seven new 
potential PDE, which had no or few biocide effect on mycelial growth and spore germination of $B$. cinerea. Using a specific bioassay, these new products were able to reduce gray mold disease development, similarly to the PDE Bion 50WG ${ }^{\circledR}$, BABA, Chitosan and MeJA. Under controlled conditions, a protective effect against $B$. cinerea was already demonstrated using BABA on Arabidopsis (Zimmerli et al. 2001; Cohen et al. 1994), Bion 50WG® on both Arabidopsis and tomato (Azami-Sardooei et al. 2013; Audenaert et al. 2002; Zimmerli et al. 2001), Chitosan on vine (Amborabe et al. 2004) and MeJA on tomato fruit (Zhu and Tian 2012; Eyre et al. 2006). To the exception of Chitosan which partly inhibited fungal germination (Fig. 2), their efficacy is not due to a direct fungicidal effect, but rather to the stimulation of plant defence reactions, leading to plant protection (Azami-Sardooei et al. 2013; Audenaert et al. 2002; Zimmerli et al. 2001; Cohen et al. 1994). Our study also characterized new potential PDE products for tomato protection against B. cinerea among which Eugenol, a phosphite, and extracts from seaweeds (Ulva spp.) and yeast (S. cerevisiae). Some of these were already characterized as plant defence elicitors in other plant/pathogen interactions, such as phosphite in Arabidopsis / Hyaloperonospora arabidopsidis (Massoud et al. 2012), and Eugenol in tomato/yellow leaf curl virus (Wang et al. 2013). Despite a good efficacy of those PDE under laboratory conditions, transfer to field conditions is sometimes found inconclusive or too variable (Small et al. 2012; Dinh et al. 2007), most probably because, in the field, many external factors can influence the efficacy of elicitor treatments. For example, protection by BTH or MeJA was shown to be ineffective in field on Geraldton waxflower (Dinh et al. 2007). To address these issues, we assayed, under controlled conditions, the impact of abiotic stresses commonly encountered during tomato growth (wounding, water stress and nitrogen deficiency) on gray mold development. First, in plants treated with water, pre-stressed plants were found to be more sensitive to $B$. cinerea than non-stressed plants. It has already been demonstrated that abiotic stresses influence the outcome of a pathogenic interaction, either negatively or positively, depending on the pathogen, timing, nature and severity of each stress (Suzuki et al. 2014; Atkinson and Urwin 2012). Some publications showed that depending on the type of pathogens, nitrogen deficiency can increase plant resistance (Stout et al. 1998; Sandermann 2004; Teng 1994; Dietrich et al. 2005). Concerning drought stress, it increases Arabidopsis susceptibility to Pseudomonas syringae (Mohr and Cahill 2003 ) but enhances resistance to B. cinerea in Arabidopsis (Chassot et al. 2008) and in tomato (Achuo et al. 2006), in contrast to our results. The discrepancy between the latter studies and our 
results may be due to the timing and intensity of the abiotic stress applied. Indeed, in Achuo et al. (2006), the drought stress was applied by suspending irrigation of 3-week-old plants until they wilted, mildly irrigating for recovery, then allowing them to wilt again three times. Plants were then inoculated with the pathogens within $18 \mathrm{~h}$ after the last recovery irrigation. In our study, pre water stressed plants were beginning to wilt and no recovery was allowed before inoculation. Therefore, it is likely that, at the time of inoculation, the magnitude of the water stress was stronger in our study than in theirs. Secondly, the protection efficacy of the products and the PDE controls was always reduced when plants were subjected to an abiotic stress prior to treatments, whatever the nature of the abiotic stress. This result suggests that the tomato responses to abiotic stresses and to treatment with the products interact negatively.

\section{AQ4}

At a molecular level, the mechanisms involved in the plant responses to combination of biotic and abiotic stresses are controlled by various mode of signalling pathways including the hormone signalling (Atkinson and Urwin 2012; Suzuki et al. 2014). Abscissic acid (ABA) and JA are the main hormones involved in response to abiotic stresses while plant signalling pathways against pests and pathogens involve mostly SA, JA, ET and ABA (Curvers et al. 2010; Kettner and Dörffling 1995; Audenaert et al. 2002; Ryan and Moura 2002; Salt et al. 1986; Ton et al. 2009). Under our experimental conditions, we found that wounding induced significant metabolic changes, including the activation of the JA pathway with a 1.1 to 3.6-fold increase for 10 oxylipins in wounded leaves 2 days after stress application and before treatment with products. It has been previously shown that JA appeared very quickly after the wounding $(<5 \mathrm{~min})$ and not only in Arabidopsis wounded leaves, but also in the leaves distal to the wounded sites in plants (Glauser 2010). Over time, the metabolic changes associated with wounding could not be discriminated from non-wounded plants, from the day of inoculation (d0) until 4 days post-inoculation. Instead, differential tomato metabolic responses were identified depending on the product treatments and fungal inoculation. In other words, the metabolic changes occurring in tomato leaves could be first attributed to wounding. Seven days after the prior abiotic stress application, the effects of wounding on metabolic regulation were then outcompeted by the effects of other external factors, the product treatment and Botrytis inoculation. Moreover, if the wounding effect on metabolic profiling disappeared 4 days after inoculation, the interaction between product 
treatment and infection with Botrytis did not lead to similar metabolic regulations (Fig. 6), suggesting a different mode of action, at least for BABA, the products $\mathrm{A}$ and $\mathrm{E}$.

More specifically, the tomato responses associated with product treatments were studied using target metabolic profiling with the time-course of two oxylipins (13HpODE and 13HOTrE), JA and SA. Interestingly, it is worth underlining that the induction of defence pathways, compared to water controls, was significantly amplified after inoculation at 4 dpi. It seems therefore that Bion $50 \mathrm{WG} \AA$, BABA, A, B, D and $\mathrm{G}$ act therefore more as priming agents than direct elicitors. In addition, we showed that treatment with products $\mathrm{A}, \mathrm{B}, \mathrm{D}$ and $\mathrm{G}$ and with Bion $50 \mathrm{WG}{ }^{\circledR}$ induced significant higher production of JA and 13-HpODE compared to water-treated controls, at 4dpi. Considering that we showed no or low direct effect of these products on the pathogen, protection efficacy conferred by these products may probably rely on their ability to induce the JA pathway, known to be involved in the resistance against necrotrophic pathogens. In addition, we also found that treatments with Bion, A, B and G led to increased SA levels at d0 and $4 \mathrm{dpi}$, with significant difference for Bion-treated leaves only detected at 0 dpi. This is not surprising given that Bion is a chemical analogue of SA and is classically used to induce SA biosynthesis in plants. The role of SA in the plant resistance against $B$. cinerea is rather complex. Using SA-deficient tomato plants, several lines of evidence indicate that SA contributes to the susceptibility of tomato plants against B. cinerea (El Oirdi et al. 2011) while a recent study supports a major involvement of the oxylipin pathway, in agreement with our study, along with a role of SA in both the basal response and the hexanoic-acid priming effect against B. cinerea (Angulo et al. 2014). Although our results cannot rule out the involvement of the SA pathway in the resistance mechanisms of tomato plants against $B$. cinerea, they underline that the activation of the JA-signalling pathway probably contribute the most to the product-induced resistant mechanisms, with the exception of BABA-induced resistant mechanisms. Indeed, under our conditions, BABA repressed or did not modulate the production of the selected metabolites except for 13-HpODE at $4 \mathrm{dpi}$, in agreement with a different action mode. Likewise, the global metabolite profiling of BABA-treated leaves was found to be significantly different compared to Bion- and water-treated plants at 4 dpi (see Online Resource, Fig. 2). BABA-induced resistant mechanisms against plant pathogens have been the focus of previous studies (Jakab et al. 2001; Zimmerli et al. 2001; Ton and Mauch-Mani 2004). In the latter study, 
the authors discovered that BABA treatment in Arabidopsis primed callose accumulation, which is controlled by an ABA-dependent defence pathway and induced resistance to two necrotrophic pathogens, Alternaria brassicicola and Plectosphaerella cucumerina. In addition, results from Zimmerli et al. (2001) also suggested that BABA enhances resistance in Arabidopsis against $B$. cinerea through potentiation of SA-dependent defence responses but not via the JA/ET signalling pathway.

AQ5

Because plant resistance to $B$. cinerea notably occurred through the activation of the JA pathway, we can wonder why, under our experimental conditions, the effects of wounding, which also induced the JA-signalling pathway, interacted negatively with the effects of the products, resulting in a lower resistance to $B$. cinerea. It is classically considered that SA can antagonize JA and vice-versa (Mur et al. 2006; Truman et al. 2007). Based on our results which indicate that SA does not seem to contribute to the product-induced resistant mechanisms against $B$. cinerea, it cannot be hypothesized that the SA-JA antagonism may account for the decreased protection efficacy of the products on pre-stressed plants. Instead, other antagonist signalling pathways may be involved that were not studied here. For example, it has been shown that plant treatments with $\mathrm{ABA}$ increase plant susceptibility to various pathogens such as B. cinerea in tomato (Audenaert et al. 2002) and $P$. syringae in Arabidopsis (Mohr and Cahill 2003). Also, the ABA-deficient tomato mutant, sitiens, was found to be more resistant to $B$. cinerea (Audenaert et al. 2002; Curvers et al. 2010). Based on these results, it can be hypothesized that the application of the abiotic stress may have also led to the activation of the ABA signaling pathway, in turn resulting in an antagonistic interaction with JA/ET signalling pathway triggered by the products.

Alternatively, hormone signalling interplay might not be the only explanation to a reduced efficacy of PDE under stressed conditions. Plant defences may be impaired after prolonged exposure to abiotic stress leading to enhanced susceptibility to subsequent biotic stresses (Suzuki et al. 2014), as suggested by the significant increase of the necrosis symptoms in stressed tomato leaves compared to unstressed controls (Fig. 3). Similarly, another study pointed that nitrogen deficiency combined with Bion $50 \mathrm{WG}{ }^{\circledR}$ treatments led to a metabolic competition between the processes involved in the defence and plant growth, resulting in a lower fitness (Heil et al. 2000). Here, the exposure of tomato plants to wounding, nitrogen deficiency or water stress has probably resulted in the increase of the metabolic costs associated with 
plant development, resulting in the weakening of plant defences and decreased efficacy of PDE.

In conclusion, we identified several promising nontoxic products and potential priming agents able to reduce gray mold development on tomato, notably through the activation of the JA pathway, but also through other signalling and regulation pathways (i.e., those involved in BABA-induced resistance). In addition, our work provides clear evidence that prior exposure of tomato plants to abiotic stress (leaf wounding, water stress or nitrogen deficiency) alters the protection efficacy of PDE treatments, suggesting an antagonistic interaction between the tomato responses to abiotic stresses and product treatments. Under our experimental conditions, we also showed that leaf cuttings and product treatments induced metabolic changes in a time-dependent manner, and that both of which activated the oxylipin and JA pathway. Interplay with other signalling events may also be involved that were not identified here, such as processes involved in plant growth or in other hormone signalling pathways. In turn, these interplays may have resulted in competitive interactions and ultimately led to an increased susceptibility of the plant to $B$. cinerea under stressed conditions. The outcomes of biotic and abiotic stress combination most probably vary according to the severity of the abiotic stress conditions, the plant species or genotype (Suzuki et al. 2014). The synergic or antagonist molecular mechanisms involved in the plant responses are also highly complex and mostly unknown (Suzuki et al. 2014). Future challenges will involve deciphering such mechanisms notably through the application of advanced omics technologies.

\section{Acknowledgment}

This work was financially supported by Vegenov-BBV, GCSAR (General Commission for Scientific Agricultural Research-Syria) and the French Ministry of Agriculture. It was also supported by companies providing the different PDE products. We are grateful to the plant pathology team in Vegenov for their technical assistance and their advices.

\section{Electronic supplementary material}

Below is the link to the electronic supplementary material. 


\section{References}

Achuo, E., Prinsen, E., \& Höfte, M. (2006). Influence of drought, salt stress and abscisic acid on the resistance of tomato to Botrytis cinerea and Oidium neolycopersici. Plant Pathology, 55(2), 178-186.

Amborabe, E., Aziz, A., Trotel-Aziz, P., Quantinet, D., Dhuicq, L., \& Vernet, G. (2004). Stimulation des défenses naturelles de la vigne: Essais d'emploi du chitosan contre Botrytis cinerea. Phytoma-La Défense des Végétaux, 571, 26-29.

Anderson, J. P., Badruzsaufari, E., Schenk, P. M., Manners, J. M., Desmond, O. J., Ehlert, C., et al. (2004). Antagonistic interaction between abscisic acid and jasmonate-ethylene signaling pathways modulates defense gene expression and disease resistance in Arabidopsis. The Plant Cell Online, 16(12), 3460-3479.

Angulo, C., De la O Leyva, M., Finiti, I., López-Cruz, J., FernándezCrespo, E., García-Agustín, P., \& González-Bosch, C. (20145). Role of dioxygenase $\alpha$-DOX2 and SA in basal response and in hexanoic acid-induced resistance of tomato (Solanum lycopersicum) plants against Botrytis cinerea. Journal of Plant Physiology, 175(2015), 163-173.

Asselbergh, B., De Vleesschauwer, D., \& Höfte, M. (2008). Global switches and fine-tuning-ABA modulates plant pathogen defense. Molecular Plant-Microbe Interactions: MPMI, 21(6), 709-719. doi: 10.1094/MPMI-21-6-0709 .

Atkinson, N. J., \& Urwin, P. E. (2012). The interaction of plant biotic and abiotic stresses: from genes to the field. Journal of Experimental Botany. doi: $10.1093 / \mathrm{jxb} / \mathrm{ers} 100$.

Audenaert, K., De Meyer, G. B., \& Höfte, M. M. (2002). Abscisic acid determines basal susceptibility of tomato to Botrytis cinerea and suppresses salicylic acid-dependent signaling mechanisms. Plant 
Azami-Sardooei, Z., Seifi, H. S., Vleesschauwer, D. D., \& Höfte, M. (2013). Benzothiadiazole (BTH)-induced resistance against Botrytis cinerea is inversely correlated with vegetative and generative growth in bean and cucumber, but not in tomato. Australasian Plant Pathology, 42(4), 485-490. doi: 10.1007/s13313-013-0207-1 .

Aziz, A., Poinssot, B., Daire, X., Adrian, M., Bézier, A., Lambert, B., et al. (2003). Laminarin elicits defense responses in grapevine and induces protection against Botrytis cinerea and Plasmopara viticola. Molecular Plant-Microbe Interactions, 16(12), 1118-1128.

Aziz, A., Trotel-Aziz, P., Dhuicq, L., Jeandet, P., Couderchet, M., \& Vernet, G. (2006). Chitosan oligomers and copper sulfate induce grapevine defense reactions and resistance to gray mold and downy mildew. Phytopathology, 96(11), 1188-1194. doi: 10.1094/PHYTO-96-1188 .

Ballester, A. R., Lafuente, M. T., Forment, J., Gadea, J., De Vos, R. C. H., Bovy, A. G., et al. (2011). Transcriptomic profiling of citrus fruit peel tissues reveals fundamental effects of phenylpropanoids and ethylene on induced resistance. Molecular Plant Pathology, 12(9), 879-897. doi: 10.1111/j.1364-3703.2011.00721.x .

Brisset, M. N., Cesbron, S., Thomson, S. V., \& Paulin, J. P. (2000). Acibenzolar-S-methyl induces the accumulation of defense-related enzymes in apple and protects from fire blight. European Journal of Plant Pathology, 106(6), 529-536.

Chassot, C., Buchala, A., Schoonbeek, H. J., et al. (2008). Wounding of Arabidopsis leaves causes a powerful but transient protection against Botrytis infection. The Plant Journal, 55(4), 555-567. doi: 10.1111/j.1365-313X.2008.03540.x

Cohen, Y., Niderman, T., Mosinger, E., \& Fluhr, R. (1994). $\beta$-Aminobutyric acid induces the accumulation of pathogenesis-related proteins in tomato (Lycopersicon esculentum L.) plants and resistance to late blight infection caused by Phytophthora infestans. Plant Physiology, 104(1), 59-66. 
Curvers, K., Seifi, H., Mouille, G., de Rycke, R., Asselbergh, B., Van Hecke, A., et al. (2010). Abscisic acid deficiency causes changes in cuticle permeability and pectin composition that influence tomato resistance to Botrytis cinerea. Plant Physiology, 154(2), 847-860. doi: 10.1104/pp.110.158972 .

De Meyer, G., \& Höfte, M. (1997). Salicylic acid produced by the rhizobacterium Pseudomonas aeruginosa 7NSK2 induces resistance to leaf infection by Botrytis cinerea on bean. Phytopathology, 87(6), 588593. AQ6

Dietrich, R., Ploss, K., \& Heil, M. (2005). Growth responses and fitness costs after induction of pathogen resistance depend on environmental conditions. Plant, Cell \& Environment, 28(2), 211-222.

Dinh, S. Q., Joyce, D. C., Irving, D. E., \& Wearing, A. H. (2007). Field applications of three different classes of known host plant defence elicitors did not suppress infection of Geraldton waxflower by Botrytis cinerea. Australasian Plant Pathology, 36(2), 142. doi: 10.1071/ap07001 .

Dufour, M. C., Lambert, C., Bouscaut, J., Mérillon, J. M., \& Corio-Costet, M. F. (2013). Benzothiadiazole-primed defence responses and enhanced differential expression of defence genes in Vitis vinifera infected with biotrophic pathogens Erysiphe necator and Plasmopara viticola. Plant Pathology, 62(2), 370-382. doi: 10.1111/j.1365-3059.2012.02628.x .

El Oirdi, M., El Rahman, T. A., Rigano, L., et al. (2011). Botrytis cinerea manipulates the antagonistic effects between immune pathways to promote disease development in tomato. The Plant Cell Online, 23(6), 2405-2421.

Eyre, J., Faragher, J., Joyce, D., \& Franz, P. (2006). Effects of postharvest methyl jasmonate treatments against Botrytis cinerea on Geraldton waxflower (Chamelaucium uncinatum). Animal Production Science, 46(5), $717-723$.

Ferrari, S., Galletti, R., Denoux, C., De Lorenzo, G., Ausubel, F. M., \& Dewdney, J. (2007). Resistance to Botrytis cinerea induced in arabidopsis by elicitors is independent of salicylic acid, ethylene, or jasmonate signaling but requires PHYTOALEXIN DEFICIENT3. Plant Physiology, 144(1), 367-379. doi: 10.1104/pp.107.095596 . 
Fu, Z. Q., \& Dong, X. (2013). Systemic acquired resistance: turning local infection into global defense. Annual Review of Plant Biology, 64, 839-863. doi: 10.1146/annurev-arplant-042811-105606 .

Glauser, G. (2010). Etude de la réponse à la blessure mécanique chez les plantes: une approche métabolomique. University of Geneva, from http://archive-ouverte.unige.ch/unige:5274 .

Heil, M., Hilpert, A., Kaiser, W., \& Linsenmair, K. E. (2000). Reduced growth and seed set following chemical induction of pathogen defence: does systemic acquired resistance (SAR) incur allocation costs? Journal of Ecology, 88(4), 645-654. doi: 10.1046/j.1365-2745.2000.00479.x .

Jakab, G., Cottier, V., Toquin, V., Rigoli, G., Zimmerli, L., Métraux, J.-P., et al. (2001). $\beta$-Aminobutyric acid-induced resistance in plants. European Journal of Plant Pathology, 107(1), 29-37.

Jones, J. D. G., \& Dangl, J. L. (2006). The plant immune system. Nature, 444(7117), 323-329. doi: 10.1038/nature05286.

Kettner, J., \& Dörffling, K. (1995). Biosynthesis and metabolism of abscisic acid in tomato leaves infected with Botrytis cinerea. Planta, 196(4), 627-634. doi: 10.1007/BF01106753.

Klarzynski, O., Plesse, B., Joubert, J. M., Yvin, J. C., Kopp, M., Kloareg, B., et al. (2000). Linear beta-1,3 glucans are elicitors of defense responses in tobacco. Plant Physiology, 124(3), 1027-1038.

Lou, B., Wang, A., Lin, C., Xu, T., \& Zheng, X. (2011). Enhancement of defense responses by oligandrin against Botrytis cinerea in tomatoes. African Journal of Biotechnology, 10(55), 442-449.

Mansfield, J. W. (1980). Mechanisms of resistance to 'Botrytis'. In J. R. Coley-Smith, K. Verhoeff, \& W. R. Jarvis (Eds.), The biology of Botrytis (pp. 181-218). New York: Academic Press.

Massoud, K., Barchietto, T., Le Rudulier, T., Pallandre, L., Didierlaurent, L., Garmier, M., et al. (2012). Dissecting phosphite-induced priming in Arabidopsis infected with Hyaloperonospora arabidopsidis. Plant Physiology, 159(1), 286-298. 
Métraux, J. P., Signer, H., Ryals, J., Ward, E., Wyss-Benz, M., Gaudin, J., et al. (1990). Increase in salicylic Acid at the onset of systemic acquired resistance in cucumber. Science (New York, N.Y.), 250(4983), 1004-1006. doi: 10.1126/science.250.4983.1004 .

Mohr, P. G., \& Cahill, D. M. (2003). Abscisic acid influences the susceptibility of Arabidopsis thaliana to Pseudomonas syringae pv. tomato and Peronospora parasitica. Functional Plant Biology, 30(4), 461-469.

Mur, L. A., Kenton, P., Atzorn, R., et al. (2006). The outcomes of concentration-specific interactions between salicylate and jasmonate signaling include synergy, antagonism, and oxidative stress leading to cell death. Plant Physiology, 140(1), 249-262.

Niinemets, Ü. (2010). Responses of forest trees to single and multiple environmental stresses from seedlings to mature plants: past stress history, stress interactions, tolerance and acclimation. Forest Ecology and Management, 260(10), 1623-1639. doi: 10.1016/j.foreco.2010.07.054 .

Pastor, V., Luna, E., Mauch-Mani, B., Ton, J., \& Flors, V. (2013). Primed plants do not forget. Environmental and Experimental Botany, 94, 46-56. doi: 10.1016/j.envexpbot.2012.02.013 .

Pozo, M. J., \& Azcón-Aguilar, C. (2007). Unraveling mycorrhiza-induced resistance. Current Opinion in Plant Biology, 10(4), 393-398. doi: 10.1016/j.pbi.2007.05.004 .

Ryan, C. A., \& Moura, D. S. (2002). Systemic wound signaling in plants: a new perception. Proceedings of the National Academy of Sciences, 99(10), 6519-6520. doi: 10.1073/pnas.112196499.

Salt, S. D., Tuzun, S., \& Kuc, J. (1986). Effects of $\beta$-ionone and abscisic acid on the growth of tobacco and resistance to blue mold. Mimicry of effects of stem infection by Peronospora tabacina Adam. Physiological and Molecular Plant Pathology, 28(2), 287-297.

Sanchez, L., Courteaux, B., Hubert, J., Kauffmann, S., Renault, J.-H., Clément, C., et al. (2012). Rhamnolipids elicit defense responses and induce disease resistance against biotrophic, hemibiotrophic, and necrotrophic pathogens that require different signaling pathways in 
Arabidopsis and highlight a central role for salicylic acid. Plant Physiology, 160(3), 1630-1641.

Sandermann, H. (2004). Molecular ecotoxicology: from man-made pollutants to multiple environmental stresses. In P. D. H. Sandermann (Ed.), Molecular ecotoxicology of plants (pp. 1-16, Ecological Studies). Berlin: Springer.

Small, I., Flett, B., Marasas, W., McLeod, A., \& Viljoen, A. (2012). Use of resistance elicitors to reduce Fusarium ear rot and fumonisin accumulation in maize. Crop Protection, 41, 10-16.

Stout, M. J., Brovont, R. A., \& Duffey, S. S. (1998). Effect of nitrogen availability on expression of constitutive and inducible chemical defenses in tomato, lycopersicon esculentum. Journal of Chemical Ecology, 24(6), 945-963. doi: 10.1023/A:1022350100718 .

Suzuki, N., Rivero, R. M., Shulaev, V., Blumwald, E., \& Mittler, R. (2014). Abiotic and biotic stress combinations. New Phytologist, 203(1), 32-43. doi: 10.1111/nph.12797 .

Teng, P. S. (1994). Epidemiological basis for blast management.

Ton, J., \& Mauch-Mani, B. (2004). $\beta$-amino-butyric acid-induced resistance against necrotrophic pathogens is based on ABA-dependent priming for callose. The Plant Journal, 38(1), 119-130. doi: 10.1111/j.1365-313X.2004.02028.x .

Ton, J., Flors, V., \& Mauch-Mani, B. (2009). The multifaceted role of ABA in disease resistance. Trends in Plant Science, 14(6), 310-317.

Trouvelot, S., Varnier, A. L., Allègre, M., Mercier, L., Baillieul, F., Arnould, C., et al. (2008). A beta-1,3 glucan sulfate induces resistance in grapevine against Plasmopara viticola through priming of defense responses, including HR-like cell death. Molecular Plant-Microbe Interactions: MPMI, 21(2), 232-243. doi: 10.1094/MPMI-21-2-0232 .

Truman, W., Bennett, M. H., Kubigsteltig, I., et al. (2007). Arabidopsis systemic immunity uses conserved defense signaling pathways and is mediated by jasmonates. Proceedings of the National Academy of Sciences 
of the United States of America, 104(3), 1075-1080. doi: 10.1073/pnas.0605423104.

Veloso, J., \& Díaz, J. (2013). Induced resistance to Botrytis cinerea in Capsicum annuum by a Fusarium crude elicitor fraction, free of proteins. Plant Biology, 15(6), 1040-1044.

Walters, D., Walsh, D., Newton, A., \& Lyon, G. (2005). Induced resistance for plant disease control: maximizing the efficacy of resistance elicitors. Phytopathology, 95(12), 1368-1373. doi: 10.1094/PHYTO-95-1368 .

Wang, Y., Loake, G. J., \& Chu, C. (2013). Cross-talk of nitric oxide and reactive oxygen species in plant programed cell death. Frontiers in Plant Science, 4, 314.

Zhu, Z., \& Tian, S. (2012). Resistant responses of tomato fruit treated with exogenous methyl jasmonate to Botrytis cinerea infection. Scientia Horticulturae, 142, 38-43.

Zimmerli, L., Métraux, J.-P., \& Mauch-Mani, B. (2001). $\beta$-Aminobutyric acid-induced protection of arabidopsis against the necrotrophic fungus Botrytis cinerea. Plant Physiology, 126(2), 517-523. doi:

10.1104/pp.126.2.5 